03

\title{
Аналитическое описание области существования тройных конфигураций с отрицательным углом наклона отраженного скачка
}

\author{
(C) М.В. Сильников, ${ }^{1,2}$ М.В. Чернышов, ${ }^{1,2, \text { Л.Г. Гвоздева }}{ }^{3}$ \\ ${ }^{1}$ Санкт-Петербургский политехнический университет Петра Великого, \\ 195251 Санкт-Петербург, Россия \\ ${ }^{2}$ НПО специальных материалов, \\ 194044 Санкт-Петербург, Россия \\ ${ }^{3}$ Объединенный институт высоких температур РАН, \\ 125412 Москва, Россия \\ ๑ e-mail: chernyshov@npo-sm.ru
}

(Поступило в Редакцию 28 декабря 2015 г. В окончательной редакции 25 апреля 2016 г.)

Рассмотрены тройные конфигурации скачков уплотнения в сверхзвуковых потоках невязкого совершенного газа. Теоретически исследованы границы области существования ударно-волновых структур нового вида (тройных конфигураций с отрицательным углом наклона отраженного скачка, или отрицательных тройных конфигураций) на множестве параметров течения.

\section{Введение}

Тройные конфигурации (ТК) скачков уплотнения или подвижных в избранной координатной системе ударных волн образуются при их взаимодействии или нерегулярном отражении [1-3]. Параметрическое исследование тройных конфигураций и свойств течения за ними, проведенное в работах [4-6], необходимо, в частности, для анализа струйных и канальных течений с маховским отражением $[7,8]$, автоколебательных режимов взаимодействия струй с преградами $[9,10]$, газодинамических явлений при работе взрывозащитных устройств [11].

Тройные конфигурации с углом наклона отраженного скачка, отрицательным по отношению к набегающему потоку („отрицательные“ ТК, или ОТК), были первоначально обнаружены экспериментально в нестационарных течениях углекислого газа, фреонов, азота и воздуха [12-16]. Численный эксперимент [17-19] показывает, что образование ОТК в стационарных течениях может привести к возникновению неустойчивостей и переходу в неустановившийся режим.

Согласно результатам параметрического анализа [17,20-22], ОТК образуются в потоках газа с малыми показателями адиабаты и большими числами Маха. Образование ОТК в двуатомных газах, по-видимому, связано с неравновесностью высокоскоростных течений, которые более адекватно описываются в рамках модели идеального газа с уменьшенным „эффективным“ показателем адиабаты.

В настоящей работе теоретически исследованы границы области существования ОТК в потоке совершенного газа. Показано, что ОТК соответствуют нерегулярному отражению скачков уплотнения, а не частным случаям взаимодействия догоняющих и встречных скачков. Аналитически найдены предельные параметры ОТК (числа
Маха, углы наклона ветвящегося скачка и показатели адиабаты газа).

\section{Общее описание области существования тройных конфигураций}

Тройные конфигурации скачков уплотнения формируются тремя скачками $(1-3$ на рис. $1, a-f)$ которые имеют общую (тройную) точку $(T)$. Газовые потоки за ТК, прошедшие разные последовательности скачков (сверху и снизу от тройной точки), разделены тангенциальным разрывом $\tau$. Параметры скачков уплотнения, формирующих конфигурацию, связаны условиями совместности на тангенциальном разрыве:

$$
J_{1} J_{2}=J_{3}, \quad \theta_{1}+\theta_{2}=\theta_{3},
$$

где $J_{1}(i=1 \ldots 3)-$ интенсивность $i$-го скачка (отношение статических давлений газа за скачком и перед ним), $\theta_{i}$ - угол поворота потока на соответствующем скачке, связанный с его интенсивностью соотношением

$$
\operatorname{tg}\left|\theta_{i}\right|=\sqrt{\frac{J_{m}-J_{i}}{J_{i}+\varepsilon}} \frac{(1-\varepsilon)\left(J_{i}-1\right)}{J_{m}+\varepsilon-(1-\varepsilon)\left(J_{i}-1\right)} .
$$

Здесь $\bar{M}_{i}$ - число Маха течения перед скачком $i$, $J_{m}\left(\bar{M}_{i}\right)=(1+\varepsilon) \bar{M}_{i}^{2}-\varepsilon-$ интенсивность прямого скачка, образующегося в потоке с соответствующим числом Маха, $\varepsilon=(\gamma-1) /(\gamma+1), \gamma-$ показатель адиабаты газа. Число Маха $M_{i}$ за скачком определяется его интенсивностью $J_{i}$ и числом Маха течения перед ним:

$$
M_{i}=\sqrt{\left[\left(J_{i}+\varepsilon\right) \bar{M}_{i}^{2}-(1-\varepsilon)\left(J_{i}^{2}-1\right)\right] /\left[J_{i}\left(\varepsilon J_{i}\right)\right]} .
$$


$a$

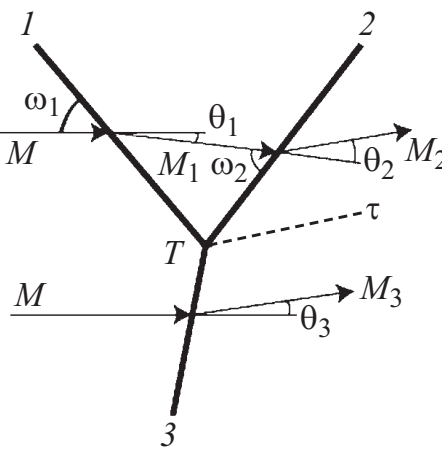

$d$

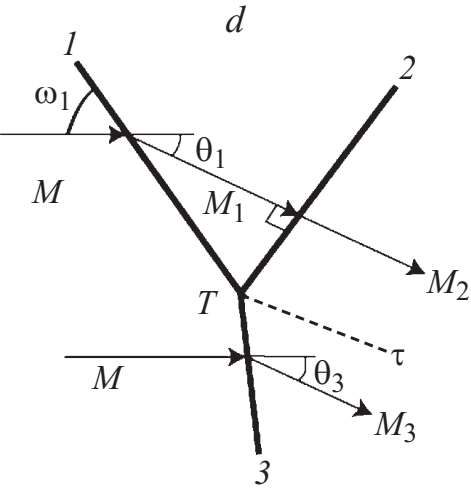

$b$

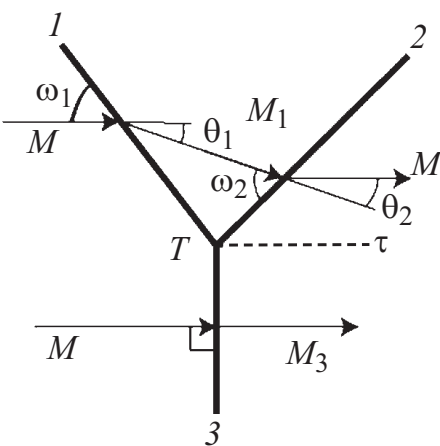

$e$

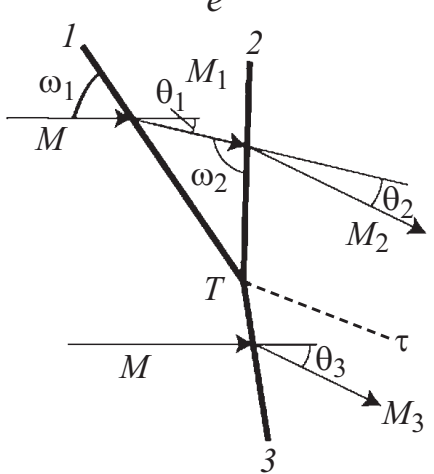

$c$

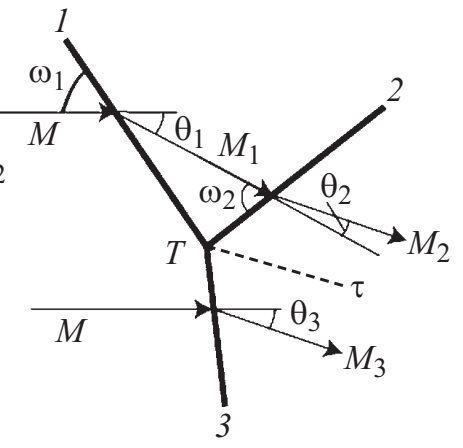

$f$

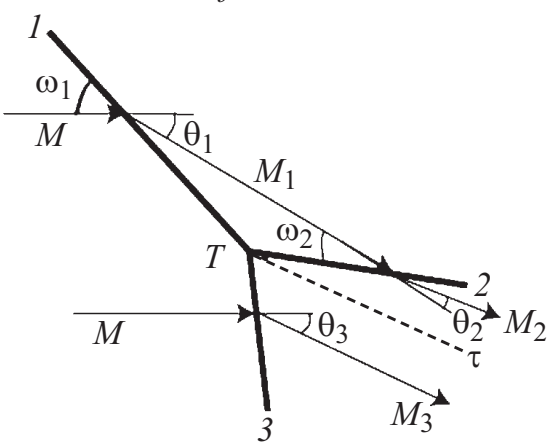

Рис. 1. Классификация тройных конфигураций скачка уплонения: $a-$ ТК-1, $b-$ СМК фон Неймана, $c-$ ТК-2, $d-$ ТКП-2-3, $e-$ TК- $3, f-$ ОТК.

В зависимости от соотношения углов $\theta_{i}$ поворота потока различают ТК первого (ТК-1, $\theta_{1} \theta_{2}<0, \theta_{1} \theta_{3}<0$, рис. $1, a)$, второго (ТК-2, $\theta_{1} \theta_{2}<0, \theta_{1} \theta_{3}>0$, рис. $1, c$ ) и третьего (ТК-3, $\theta_{1} \theta_{2}<0, \theta_{1} \theta_{3}>0$, рис. $1, e$ ) типов. Стационарная маховская конфигурация СМК $\left(\theta_{3}=0\right.$, рис. $1, b)$, соответствующая критерию фон Неймана смены типа отражения скачков уплотнения, является переходной между структурами ТК-1 и ТК-2, а переходная конфигурация ТКП $\left(\theta_{2}=0\right.$, рис. $\left.1, d\right)$ - между ТК-2 и ТК-3. На рис. $1, f$ показаны ОТК $\left(\theta_{1}>\omega_{2}\right.$, что приводит к отрицательному наклону скачка 2 по отношению к невозмущенному потоку), которые и рассматриваются в дальнейшем.

Угол $\omega_{i}$ наклона поверхности скачка к вектору скорости потока перед ним определяется интенсивностью соответствующего скачка и числом Маха течения перед ним:

$$
\sin \omega_{i}=\sqrt{\left(J_{i}+\varepsilon\right) /\left[(1+\varepsilon) \bar{M}_{i}^{2}\right]} .
$$

Для решения (иногда не единственного) задачи (1)-(4) расчета параметров всех скачков ТК и течения за ними достаточно определить число Маха $M$ невозмущенного потока, интенсивность $J_{1}$ скачка 1 (или его угол наклона $\left.\omega_{1}\right)$, а также показатель адиабаты газа. При фиксированном значении показателя адиабаты параметрический анализ ТК, как правило, проводится на плоскости $(M, \omega)$, представленной на рис. 2.

Кривая $1\left(\omega_{1}=\mu(M)\right.$, где $\mu(M)=\arcsin (1 / M)-$ угол Маха) служит нижней границей области существования ТК. Кривые $2 a-2 d$, которые соответствуют смене типа отраженного возмущения в задаче о взаи-

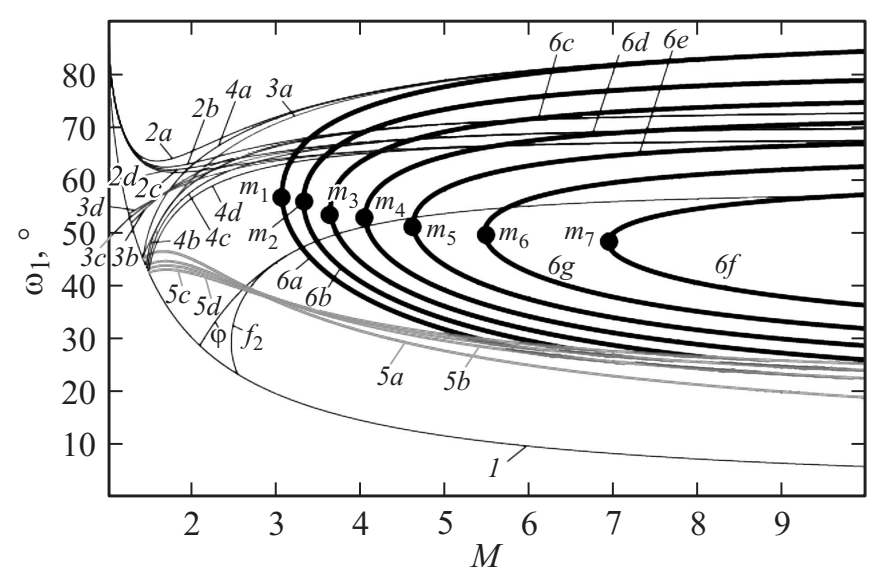

Рис. 2. Область существования ОТК на плоскости „число Маха потока - угол наклона ветвящегося скачка“. Кривые $2 a-2 d-5 a-5 d$ соответствуют показателям адиабаты газа $\gamma=1,1.2,1.3,1.4$, кривые $6 a-6 g-$ значениям $\gamma=1,1.05,1.1,1.15,1.2,1.25$ и 1.3 . 
модействии косого скачка с последующим догоняющим слабым разрывом и определяются уравнением $[4,22]$

$$
\begin{gathered}
M=\sqrt{[A(B-C)-1+\varepsilon] / \varepsilon} \\
A=\left(1+\varepsilon J_{1}\right) /\left[(1+\varepsilon)\left(J_{1}(1-3 \varepsilon)-4 \varepsilon^{2}\right)\right], \\
B=J_{1}\left(1-2 \varepsilon-\varepsilon^{2}\right)-2 \varepsilon^{2}, \\
C=2 \varepsilon \sqrt{\varepsilon\left(1+\varepsilon J_{1}\right)\left(J_{1}+\varepsilon\right)},
\end{gathered}
$$

являются верхней границей области существования при соответствующем значении показателя адиабаты. Для существования ТК необходим сверхзвуковой характер течения за скачком 1, но это условие не является достаточным - кривые $3 a-3 d$, определяемые условием $M_{1}=1$, расположены выше области существования ТК, в действительности ограниченной кривыми 1 и $2 a-2 d$.

Кривые $4 a-4 d$ и $5 a-5 d$, соответствующие образованию переходных конфигураций с прямыми скачками 3 и 2 (СМК и ТКП соответственно), разделяют зону между кривыми 1 и $2 a-2 d$ на области I-III существования тройных конфигураций соответствующих типов. Стационарная маховская конфигурация СМК (кривые $4 a-4 d$ ) определяется соотношением

$$
\begin{gathered}
M^{2}=\left(a_{1}+\sqrt{a_{1}^{2}-4 a_{0} a_{2}}\right) / 2 a_{2}, \\
a_{2}=\left(1-\varepsilon^{2}\right)\left(1+\varepsilon J_{1}\right), \\
a_{1}=\left(1+\varepsilon-\varepsilon^{2}+\varepsilon^{3}\right) J_{1}^{2}+\varepsilon\left(1+3 \varepsilon+2 \varepsilon^{2}\right) J_{1} \\
-(1-\varepsilon)(1+2 \varepsilon), \\
a_{0}=(1-\varepsilon)\left(J_{1}-1\right)\left(J_{1}^{2}+\varepsilon(1-\varepsilon) J_{1}-\varepsilon\right),
\end{gathered}
$$

а переходная между ТК-2 и ТК-3 конфигурация ТКП (кривые $5 a-5 d)$ - уравнением

$$
\begin{aligned}
& M^{4}-r M^{2}+\left(J_{1}-1\right)\left(J_{1}+2-\varepsilon\right) /(1+\varepsilon)=0, \\
r= & \left(J_{1}-1\right)\left(J_{1}+2-\varepsilon\right) /\left(J_{1}+\varepsilon\right)+\left(J_{1}+\varepsilon\right) /(1+\varepsilon) \\
& +\left(1+\varepsilon J_{1}\right)^{2} /\left[(1-\varepsilon)\left(J_{1}+\varepsilon\right)^{2}\right] .
\end{aligned}
$$

В области, ограниченной кривой 1 и линиями $\varphi$ и $f_{2}$, построенными для примера при значении $\gamma=1.4$, описанное решение системы (1)-(4), включающее тройные конфигурации всех трех видов, не является единственным. При тех же значениях параметров $M$ и $\omega_{1}$ присутствуют еще два (в криволинейном треугольнике) или одно (в зоне между кривыми 1 и $f_{2}$ ) решение, описывающее конфигурацию третьего типа со сверхзвуковым течением за всеми скачками уплотнения. Эти „альтернативные“ [4] конфигурации не относятся к ОТК и из дальнейшего рассмотрения исключаются.

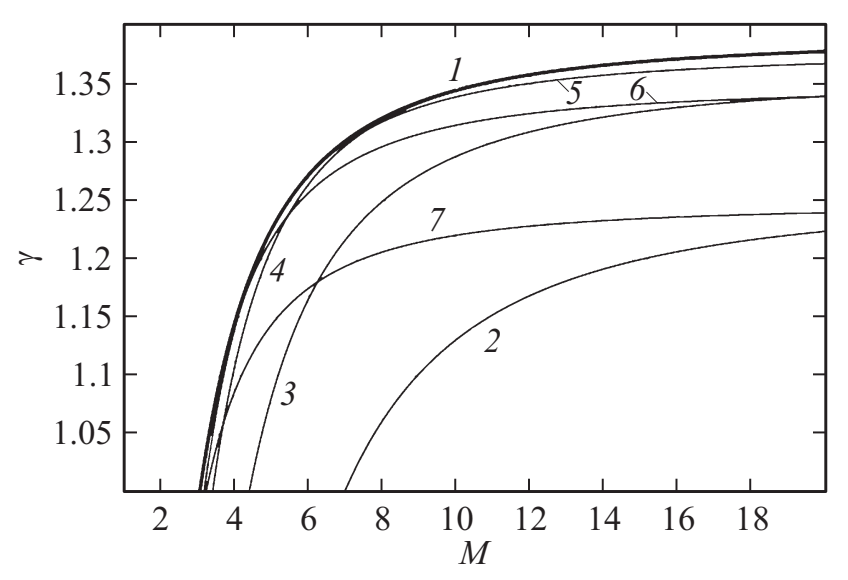

Рис. 3. Минимальный числа Маха, соответствующие образованию ОТК при различных значениях показателя адиабаты газа (кривая 1). Границы области существования ОТК при различных фиксированных углах наклона ветвящегося скачка: $\omega_{1}=25,35,45,50,55$ и $65^{\circ}$ (кривые $2-7$ ).

\section{Анализ области существования ОTK}

Преобразования системы (1)-(4), описывающей тройные конфигурации в целом, приводят к алгебраическим уравнениям высоких степеней, которые связывают число Маха течения с интенсивностями скачков 1 и 2 или тригонометрическими функциями их углов наклона $[17,23]$. Дополнительное условие

$$
\omega_{2}=\left|\theta_{1}\right|
$$

определяет границы области существования ОТК при различных показателях адиабаты (кривые $6 a-6 g$ на рис. 2).

Максимальный критический (при $M \rightarrow \infty$ и $M_{1} \rightarrow 1$ ) угол $\theta_{1}$ поворота потока на скачке 1 , равный $\arcsin (1 / \sqrt{1+\varepsilon})$, не превосходит $90^{\circ}$. По этой причине ОТК не могут образоваться при взаимодействии догоняющих скачков $\left(\omega_{2}>90\right.$ в ТК-3, см. рис. $\left.1, e\right)$. Суммарный угол $\theta_{3}=\theta_{1}+\theta_{2}$ поворота потока в ОТК отрицателен (рис. $1, f)$, что исключает образование ОТК при взаимодействии встречных скачков $\left(\theta_{3}>0\right.$ в ТК-1, рис. $\left.1, a\right)$. Следовательно, ОТК образуются при маховском отражении скачков уплотнения и относятся к виду ТК-2, а их область существования ограничена семействами кривых 4 (СМК) и 5 (ТКП).

Как отмечалось ранее, ОТК возникают при умеренных и больших числах Маха. Наименьшее число Маха $(M=3.064)$, при котором существует решение системы (1)-(5), соответствует предельному случаю $(\gamma \rightarrow 1)$ и следующим параметрам падающего скачка 1 : $J_{1}=6.626, \omega_{1}=57.144^{\circ}$ (точке $m_{1}$ на рис. 2 ). Наименьшие числа Маха, соответствующие образованию ОТК при других показателях адиабаты газа, отмечены на рис. 2 точками $m_{2}-m_{1}$, а также показаны на рис. 3 (кривая 1 ). Значение угла $\omega_{1}$ наклона ветвящегося скачка на кривой 1 переменно: от 57.144 до $44.605^{\circ}$. Кривые $2-7$ 
являются верхними границами области существования ОТК на плоскости $(M, \gamma)$ при различных фиксированных значениях угла $\omega_{1}$.

Дальнейшее увеличение показателя адиабаты газа приводит к вытеснению области существования ОТК в промежуток крайне больших чисел Маха (в частности, при $\gamma=1.35$ ОТК образуются в диапазоне $M>10.598$, а если $\gamma=1.39$ - лишь при $M>274.963)$. При $\gamma>1.392$ физически обоснованные (с действительными значениями интенсивностей скачков, превышающими единицу) решения системы (1)-(5) отсутствуют. Таким образом, ОТК теоретически нереализуемы в идеальном одно- или двуатомном газе.

При больших числах Маха границы областей существования ОТК имеют горизонтальные асимптоты, описываемые соотношениями

$$
J_{1} / M^{2} \rightarrow C \quad \text { или } \quad \omega_{1} \rightarrow \arcsin \sqrt{C /(1+\varepsilon)} .
$$

Значения коэффициента $C$, соответствующие нижней и верхней асимптотам, представляют собой два расположенные на отрезке $[0 ; 1]$ корня уравнения

$$
\begin{gathered}
\sum_{k=0}^{9} c_{k} C^{k}=0 \\
c_{9}=4(1-\varepsilon)^{9}, \quad c_{8}=-4\left(7-10 \varepsilon+\varepsilon^{2}\right)\left(1-\varepsilon^{2}\right), \\
c_{7}=\left(85-230 \varepsilon+180 \varepsilon^{2}-30 \varepsilon^{3}-\varepsilon^{4}\right)(1+\varepsilon) \cdot \gamma, \\
c_{6}=-\left(146-261 \varepsilon+86 \varepsilon^{2}+9 \varepsilon^{3}\right)(1+\varepsilon)^{2} \cdot \gamma, \\
c_{5}=\left(155-559 \varepsilon+722 \varepsilon^{2}-372 \varepsilon^{3}+27 \varepsilon^{4}+31 \varepsilon^{5}\right) \cdot \gamma^{4}, \\
c_{4}=-\left(104-213 \varepsilon+87 \varepsilon^{2}+68 \varepsilon^{3}-52 \varepsilon^{4}\right)(1+\varepsilon) \cdot \gamma^{4}, \\
c_{3}=\left(43-81 \varepsilon-27 \varepsilon^{2}+187 \varepsilon^{3}-179 \varepsilon^{4}+45 \varepsilon^{5}\right) \cdot \gamma^{6}, \\
c_{2}=-\left(10+5 \varepsilon-65 \varepsilon^{2}+109 \varepsilon^{3}-79 \varepsilon^{4}+18 \varepsilon^{5}\right) \cdot \gamma^{7}, \\
c_{1}=\left(1+7 \varepsilon-17 \varepsilon^{2}+19 \varepsilon^{3}-4 \varepsilon^{4}\right) \cdot \gamma^{8}, \\
c_{0}=-\varepsilon\left(1-\varepsilon+2 \varepsilon^{2}\right) \cdot \gamma^{9}
\end{gathered}
$$

За исключением предельного случая $(C \rightarrow 0$ для нижней и $C \rightarrow 1$ для верхней асимптоты при $\gamma \rightarrow 1$ ), асимптоты границ областей существования ОТК лежат между горизонтальными асимптотами границ областей существования тройных конфигураций второго типа. Как известно из [4], нижняя граница области существования ТК-2, соответствующая СМК фон Неймана, при $M \rightarrow \infty$ имеет горизонтальную асимптоту

$$
\frac{J_{1}}{M^{2}} \rightarrow \frac{2 \varepsilon\left(1-\varepsilon^{2}\right)}{1+\varepsilon-\varepsilon^{2}+\varepsilon^{3}+\sqrt{D}}
$$

или

$$
\begin{gathered}
\omega_{1} \rightarrow \arcsin \sqrt{\frac{2 \varepsilon(1-\varepsilon)}{1+\varepsilon-\varepsilon^{2}+\varepsilon^{3}+\sqrt{D}}}, \\
D=(1+\varepsilon)^{2}-\varepsilon(1-\varepsilon)\left[2(1+\varepsilon)(2-\varepsilon)-\varepsilon^{3}(1-\varepsilon)\right],
\end{gathered}
$$

а верхняя граница, соответствующая ТКП - асимптоту вида

$$
J_{1} / M^{2} \rightarrow 1 \quad \text { или } \quad \omega_{1} \rightarrow \arcsin \sqrt{1 /(1+\varepsilon)} .
$$

Совпадение корней и экстремумов многочлена в левой части уравнения (6) на промежутке $C \in[0 ; 1]$ определяет предельный показатель адиабаты газа, при котором существуют ОТК, и соответствующие этому значению $\gamma$ параметры тройных конфигураций. Как показывает анализ решений уравнения (6), при $\gamma \rightarrow 1.392$ верхняя и нижняя асимптоты границ областей существования ОТК совпадают $\left(C \rightarrow 0.574, \omega_{1} \rightarrow 44.605^{\circ}\right)$, и при больших значениях показателя адиабаты газа соответствующие корни уравнения (6) отсутствуют. Значение предельного показателя адиабаты $(\gamma=1.392)-$ единственное расположенное на промежутке $[1 ; 2]$ из двенадцати вещественных корней следующего уравнения:

$$
\begin{aligned}
& 370 \gamma^{34}+5424 \gamma^{33}-175854 \gamma^{32}-886784 \gamma^{31} \\
& +13026646 \gamma^{30}+81408875 \gamma^{29}-47569289 \gamma^{28} \\
& -764585958 \gamma^{27}+435297096 \gamma^{26}+2955453411 \gamma^{25} \\
& -5531543817 \gamma^{24}+2922651368 \gamma^{23}+23552398286 \gamma^{22} \\
& -78528706438 \gamma^{21}+13388896772 \gamma^{20} \\
& +297234789943 \gamma^{19}-405949888557 \gamma^{18} \\
& -322812747109 \gamma^{17}+1261998910539 \gamma^{16} \\
& -715547823068 \gamma^{15}-1364377023114 \gamma^{14} \\
& +2400402109703 \gamma^{13}-602590771313 \gamma^{12} \\
& -2132314914708 \gamma^{11}+2552815190602 \gamma^{10} \\
& -344714782135 \gamma^{9}-1644727209987 \gamma^{8} \\
& +1449571196988 \gamma^{7}-176634128738 \gamma^{6} \\
& -454615163124 \gamma^{5}+335896060478 \gamma^{4} \\
& -108536520069 \gamma^{3}+17665892209 \gamma^{2} \\
& -1256563311 \gamma+15945191=0 .
\end{aligned}
$$

\section{Заключение}

ОТК относятся к виду ТК-2, соответствующему нерегулярному отражению скачков, а не частным случаям их догоняющего или встречного взаимодействия. Они образуются в установившихся течениях при больших числах Маха $(M>3.064)$ и в широком диапазоне значений показателя адиабаты газа $(\gamma \leq 1.392)$; однако числа Маха, при которых их возникновение теоретически возможно, крайне велики при $\gamma>1.35$. Границы области существования ОТК имеют горизонтальные асимптоты, уравнения которых зависят от значения показателя адиабаты, на плоскости число Маха потока - угол наклона ветвящегося скачка. 
Работа была выполнена при финансовой поддержке Российского фонда фундаментальных исследований (проект 14-08-01070).

\section{Список литературы}

[1] von Neumann J. Oblique reflection of shock waves. Collected Works. London: Pergamon Press, 1963. Vol. 6. P. 238-299.

[2] Ландау Л.Д., Лифиии, Е.М. Теоретическая физика. Т. VI. Гидродинамика. М.: Наука, 1986. 736 с.

[3] Омельченко А.В., Усков В.Н. // Письма в ЖТФ. 2002. Т. 28. Вып. 12. C. 5-12.

[4] Усков В.Н., Чернышов М.В. // Прикладная механика и техническая физика. 2006. Т. 47. № 4. С. 39-53.

[5] Hekiri H., Emanuel G. // Shock Waves. 2011. Vol. 21. N 6. P. 511-521.

[6] Hekiri H., Emanuel G. // Phys. Fluid. 2015. Vol. 27. N 5. P. 056102.

[7] Омельченко А.В., Усков В.Н., Чернышов М.В. // Письма в ЖТФ. 2003. Т. 29. Вып. 6. С. 56-62.

[8] Ivanov M.S., Ben-Dor G., Elperin T., Kudryavtsev A.N., Khotyanovsky D.V. // J. Fluid Mechanic. 2002. Vol. 469. P. $71-87$.

[9] Гинзбург И.П., Семилетенко Б.Г., Терпигорьев В.С., Усков В.Н. // Инженерно-физический журнал. 1970. Т. 19. № 3. C. 412-417.

[10] Kim S.I., Park S.O. // Shock Waves. 2005. Vol. 14. N 4. P. 259-272.

[11] Gelfand B.E., Silnikov M.V., Chernyshov M.V. // Shock Waves. 2010. Vol. 20. N 4. P. 317-321.

[12] Гвоздева ЛІ.Г., Предводителева О.А. // Докл. АН СССР. 1965. T. 163. № 5. C. 1088-1091.

[13] Бажсенова Т.В., Гвоздева Л.Г. Нестационарные взаимодействия ударных волн. М.: Наука, 1977. 274 с.

[14] Bazhenova T.V., Gvozdeva L.G., Nettleton M.A. // Progr. in Aerospac. Sci. 1984. Vol. 21. N C. P. 249-331.

[15] Semenov A.V., Berezkina M.K., Krassovskaya I.V. // Shock Waves. 2012. Vol. 22. N 4. P. 307-316.

[16] Березкина М.К., Сыщикова М.П., Семенов А.Н. // ЖТФ. 1982. Т. 32. Вып. 7. С. 1375-1385.

[17] Gvozdeva L.G., Silnikov M.V., Gavrenkov S.A. // Acta Astronautica. 2015. Vol. 116. P. 36-42.

[18] Gvozdeva L.G., Gavrenkov S.A. A new configuration of irregular reflection of shock waves. Proceedings of the $6^{\text {th }}$ European Conf. for Aeronautics and Space Sciences (EUCASS). Krakov, 2015. Vol. 7. P. 437-452.

[19] Гавренков С.А., Гвоздева Л.Г. // Письма в ЖТФ. 2012. Т. 38. Вып. 12. С. 74-80.

[20] Гвоздева Л.Г., Гавренков С.А. // Письма в ЖТФ. 2012. Т. 38. Вып. 8. С. 39-45.

[21] Гвоздева Л.Г., Гавренков С.А. // ЖТФ. 2013. Т. 83. Вып. 8. C. $155-158$.

[22] Gvozdeva L.G., Chulyunin A.Yu. Numerical investigation of regular and Mach reflection at the expiration of the gas jet from the nozzle. Proceedings of the $6^{\text {th }}$ European Conf. for Aeronautics and Space Sciences (EUCASS). Krakov, 2015. $9 \mathrm{p}$.

[23] Усков В.Н., Чернышов М.В. // Теплофизика и аэромеханика. 2014. Т. 21. № 1. С. 15-31.

[24] Henderson L.F. // The Aeronautical Quarterly. 1964. Vol. 15. P. 181-197. 